\title{
Interfacial tension of aqueous biopolymer mixtures close to the critical point
}

\author{
Y.A. Antonov, P. Van Puyvelde*, P. Moldenaers \\ Department of Chemical Engineering, Katholieke Universiteit Leuven, W. de Croylaan 46, B-3001 Leuven, Belgium
}

Received 17 December 2003

\begin{abstract}
Proteins and polysaccharides, being the main constructional materials in many biological structures, have a limited compatibility in aqueous media. At sufficiently high concentrations, they form water-in-water emulsions. Interfacial tension is an important parameter in such systems since it is a controlling factor in the morphology development during processing. In this work a rheo-optical methodology, based on the analysis of small angle light scattering (SALS) patterns during fibril break-up, is used to study the interfacial tension of water-sodium caseinate-sodium alginate systems located close to and relatively far from the binodal. The interfacial tension close to the critical point was $\sim 10^{-8} \mathrm{~N} / \mathrm{m}$, and it increased considerably, to a value of up to $5.2 \times 10^{-6} \mathrm{~N} / \mathrm{m}$ farther from the critical point. For the scaling of the interfacial tension with the density difference between the phases, a scaling exponent of $3.1 \pm 0.3$ was found, in agreement with the critical mean-field scaling exponent of 3.
\end{abstract}

(C) 2004 Published by Elsevier B.V.

Keywords: Biopolymer mixtures; Interfacial tension; Rheo-optical methodology; Light scattering; Blends

\section{Introduction}

Biopolymer mixtures are of importance to control the composition-structure-property relationship in natural biological systems and in formulated foods [1-4]. The enormous diversity of food and other biological structures is based on only two kinds of macromolecules: proteins and polysaccharides, which are the most important constructional materials [5]. Water is a good solvent for each biopolymer, and it is known that their mixtures can display aggregative or segregative phase behaviour. In the latter situation, each phase becomes enriched in either polysaccharides or proteins. Liquid-liquid phase separation in biopolymer mixtures is the rule, but it occurs at relatively high concentrations and at particular physico-chemical conditions depending on the polyelectrolytic properties and the self-association behaviour of the mixed macromolecules $[6,7]$. The term 'water-in-water emulsion' was introduced to distinguish them from oil-in-water and water-in-oil emulsions [5]. These water-in-water emulsions are characterised by a very low interfacial tension $[1,8-10]$. During the last 10

\footnotetext{
* Corresponding author. Tel.: +32-16-322357; fax: +32-16-322991.

E-mail address: peter.vanpuyvelde@cit.kuleuven.ac.be (P. Van Puyvelde).
}

years, a significant amount of experimental (e.g. [11,12]) and theoretical studies (e.g. $[13,14]$ ) have been performed to clarify the conditions of the incompatibility, to establish the origin of the interactions that lead to phase separation and even to predict phase diagrams. The main results of these studies were summarised in a number of reviews (e.g. $[1,15,16])$.

If phase separation takes place, the driving force for decomposition must overcome the accompanying increase in interfacial free energy, which equals the product of the interfacial tension and the total interfacial area associated with the phase separation. Therefore the interfacial tension between the coexisting phases is of fundamental interest. In general, interfacial tension is of scientific and practical importance due to its relation to the thermodynamic state of biopolymer interfaces, its role in phase separation processes [17] as well as due to its relation to the morphology development in liquid two-phase fluid-fluid mixtures [18]. The interfacial tension in aqueous biopolymer protein-polysaccharide mixtures is, in contrast to the phase equilibrium, an aspect to which limited attention has yet been paid. The magnitude of the interfacial tension depends on the position of the system on the phase diagram, tending to zero as the critical point is approached. Stokes et al. [19] applied shear to a two-phase gelatin-maltodextrin system, and from the shape of the droplets, they estimated an interfacial tension to be in 
the range of $(50-100) \times 10^{-6} \mathrm{~N} / \mathrm{m}$. Recently Guido et al. [20] developed an experimental methodology based on the analysis of the shape of a single droplet and reported very low interfacial tensions, of the order $10^{-5}$ to $10^{-6} \mathrm{~N} / \mathrm{m}$ for sodium alginate-sodium caseinate systems. Van Puyvelde et al. [9] applied a rheo-optical methodology, based on small angle light scattering (SALS), to measure the interfacial tension of an aqueous gelatin-dextran system located far from the critical point. They obtained a value of the order of $10^{-5} \mathrm{~N} / \mathrm{m}$. Recently Scholten et al. evaluated the interfacial tension of gelatin-dextran-1 M NaI systems located far from and close to the critical point by the spinning drop method [10]. The value obtained was less than $10^{-6} \mathrm{~N} / \mathrm{m}$ and it increased, in a non-linear manner, farther away from the critical point. It should be noted that a highly concentrated $\mathrm{NaI}$ solution was used as a solvent to suppress the gelation of gelatin and to achieve small density differences between the coexisting phases.

The aim of this work is to determine the values of the interfacial tension in a two-phasic water-protein-polysaccharide mixture close to its critical condition, i.e. at phase compositions as close as possible to the critical point. The distance with respect to the critical point is expressed as the density difference between the coexisting phases, since at the critical point, this density difference becomes zero. A rheo-optical methodology [21,22] based on the analysis of the SALS patterns during fibril break-up was used.

This method already proved to be well suited for measuring the interfacial tension in aqueous biopolymer mixtures [9]. Here it will be explored how far the method can be extended to obtain values of the interfacial tension as close as possible to the critical point. In addition, a scaling relation for the interfacial tension will be presented that allows an estimate of the interfacial tension once the densities of the coexisting phases are known.

\section{Materials and methods}

The experiments are performed on a water-sodium caseinate-sodium alginate $(\mathrm{w}-\mathrm{c}-\mathrm{a})$ system. These materials have been chosen because of their relatively large optical density which provides a large contrast for the light scattering experiment. Alginate is an anionic polysaccharide consisting of linear chains of (1-4)-linked $\beta$-D-mannuronic and $\alpha$-L-guluronic acid residues. These residues are arranged in blocks of mannuronic or guluronic acid residues linked by blocks in which the sequence of the two acid residues is predominantly alternating [23]. Casein is a protein composed of a heterogeneous group of phosphoproteins organised in micelles. Sodium caseinate is a salt of casein, obtained by disrupting the supra-molecular organisation. This means that the polymer is a random coil and that the internal structure of the micelles is lost. The isoelectric point is around $\mathrm{pH}=4.7-5.2$ [24]. The caseinate at neutral $\mathrm{pH}$ is thus negatively charged, like alginate. Both biopolymers are well known, widely used in industry, and the thermodynamic behaviour of the ternary water-caseinate-alginate systems is known from literature [6,25-28].

The sodium caseinate sample (90\% protein, $5.5 \%$ water content, $3.8 \%$ ash, $0.02 \%$ calcium) was purchased from Sigma Chemical Co. The weight average molecular mass of the sodium caseinate is $320 \mathrm{kDa}$. The medium viscosity sodium alginate, extracted from brown seaweed (Macrocystis pirifera), was purchased from Sigma. The molecular weight of the sample, as reported in literature, was $390 \mathrm{kDa}$ [20]. Solutions of sodium caseinate and alginate were prepared by dispersing biopolymers in bi-distilled water under stirring and by heating for $1 \mathrm{~h}$ at $318 \mathrm{~K}$. The solutions were then cooled to $298 \mathrm{~K}$ and stirred again for $1 \mathrm{~h}$. The required $\mathrm{pH}$ values of solutions (7.0) were adjusted by addition of $0.1-0.5 \mathrm{M} \mathrm{NaOH}$ or $\mathrm{HCl}$. The resulting solutions were centrifuged at $60,000 \times g$ for $1 \mathrm{~h}$ at $298 \mathrm{~K}$ to remove insoluble particles. Concentrations of the solutions are determined by drying at $373 \mathrm{~K}$ up to constant weight. The ternary water-caseinate-alginate systems with required compositions were prepared by mixing solutions of each biopolymer at $298 \mathrm{~K}$. After mixing for $1 \mathrm{~h}$, the systems were centrifuged at $60,000 \times g$ for $1 \mathrm{~h}$ at $298 \mathrm{~K}$ to separate the phases using a temperature-controlled rotor.

The phase diagram of the ternary systems was obtained at $\mathrm{pH}$ 7.0, and $298 \mathrm{~K}$ as illustrated schematically in Fig. 1a and $b$. The procedure is adapted from Koningsveld and Staverman [29] and Polyakov et al. [30]. The separately prepared protein and polysaccharide solutions (concentrations $C_{2}^{\mathrm{o}}$ and $C_{3}^{\mathrm{o}}$, respectively) were mixed at $298 \mathrm{~K}$ in various weight ratios, yielding mixtures with protein and polysaccharide concentrations $C_{2}$ and $C_{3}$ (wt.\%), respectively, which are represented on the phase diagram by the secant $\left[C_{2}^{\mathrm{o}}, C_{3}^{\mathrm{o}}\right]$. The phase state of the systems was determined by eye after centrifugation $(60,000 \times g$ for $1 \mathrm{~h}$ at $298 \mathrm{~K})$ following a rest period of $1 \mathrm{~h}$ at $298 \mathrm{~K}$. When phase separation occurred, the volumes of the two coexisting liquid phases were determined by weighing, assuming the density of the phases to be equal to 1.0. The points where the binodal intersects the secant $\left[C_{2}^{\mathrm{o}}, C_{3}^{\mathrm{o}}\right]$ are given by extrapolation of the relation: $r=V^{\prime} /\left(V^{\prime}+V^{\prime \prime}\right)=f\left(C_{2} / C_{2}^{0}\right)$ to $r=0$ and 1 . $V^{\prime}$ and $V^{\prime \prime}$ represent volumes of the protein-enriched (lower phase) and the polysaccharide-enriched (upper phase) phases, respectively. The value $r=0.5$ gives the position of the middle of the tie lines. By repeating this procedure for a series of values of $C_{2}^{\mathrm{o}}$ and $C_{3}^{\mathrm{o}}$, the phase diagram can be reconstructed from the set of points corresponding to the binodal and the centre of the tie lines. The phase composition of the separated systems was derived from both the material balance of the systems studied and the position of the binodal in the plot, using the correlation [31]:

$\frac{V^{\prime \prime}}{V^{\prime}}=\frac{\rho^{\prime} X}{\rho^{\prime \prime} Y}$

where $V^{\prime \prime}, V^{\prime}$ and $\rho^{\prime \prime}, \rho^{\prime}$ are the volumes and density of the polysaccharide enriched and casein-enriched phases, 


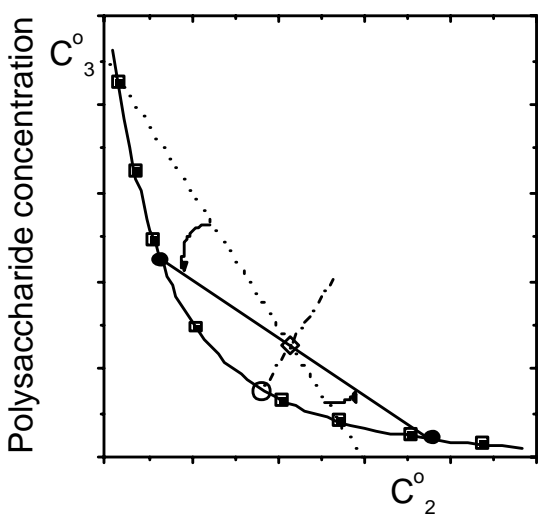

(a)

Protein concentration

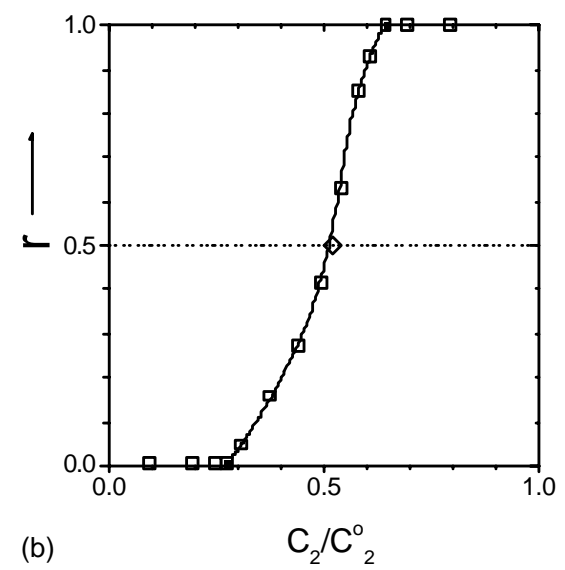

Fig. 1. Schematic illustration of the phase volume ratio method. (a) Fragment of the phase diagram: dash-dotted line, secant; thick full line, binodal; thin full line, tie line; $\boldsymbol{O}$, phase composition; $\mathbf{\square}$, points of the binodal; $\bigcirc$, critical point; dotted line, rectilinear diameter. (b) Typical dependence of the phase volume ratio on mixture composition.

respectively. $X$ and $Y$ are the distances in the plot between the points corresponding to (i) the composition of protein-enriched phase and the composition of initial mixture $(X)$, and (ii) the composition of polysaccharide-enriched phase and the composition of the initial mixture $(Y)$. The phase composition of the separated systems was checked on the basis of the system's material balance. The threshold point was determined from the plot as the point where the line with the slope -1 is tangent to the binodal. The critical point of the system was defined as the point where the binodal intersects the rectilinear diameter, which is the line joining the centre of the tie lines (Fig. 1).

The resulting isothermal phase diagram of the $\mathrm{w}-\mathrm{c}-\mathrm{a}$ system is presented in Fig. 2, both in the Cartesian as well as in the classical triangular coordinates. The phase diagram was constructed to determine the critical point and is required to study the density difference and concentration dependence of the interfacial tension. Due to the high content of water in the coexisting phases and the strong difference in solubil-

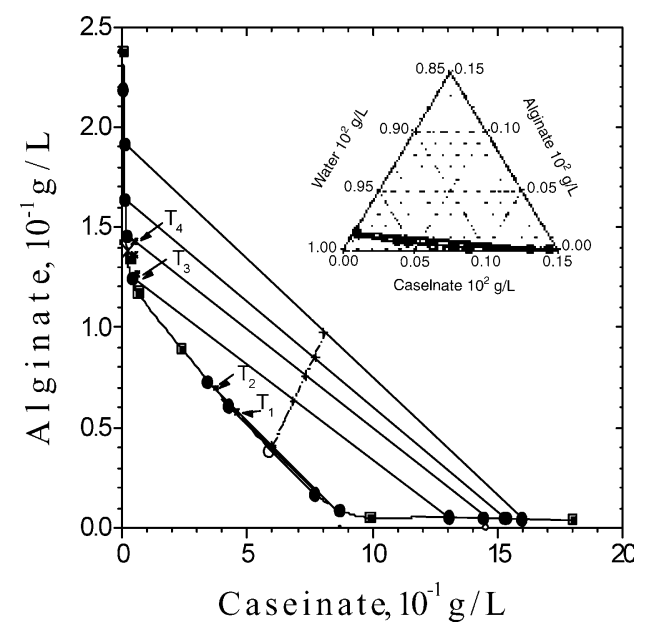

Fig. 2. Isothermal phase diagram of the water-sodium caseinate-sodium alginate system $(\mathrm{pH} 7.0,298 \mathrm{~K})$. Coordinates of the critical point: caseinate, $5.91 \%$; alginate, $0.38 \%$. ity, the phase diagram plotted in the Cartesian co-ordinates is more informative then the classical triangular representation. The phase diagram is characterised by low values of the total threshold concentration $\left(C_{\mathrm{t}}^{*}=16 \mathrm{~g} / \mathrm{l}\right)$, a relatively high total concentration of biopolymers at the critical point $\left(C_{\mathrm{c}, \mathrm{t}}=62.9 \mathrm{~g} / \mathrm{l}\right)$ and a strong asymmetry $\left(K_{\mathrm{s}}=15.5\right)$.

The viscosities of the alginate-enriched phases have been measured at $298 \mathrm{~K}$ on a stress-controlled instrument (DSR, Rheometric Scientific) equipped with a Couette geometry. The viscosities of the caseinate-enriched phases have been determined with a Ubelhode viscometer at the same temperature. Gay-Lussac pyknometers have been used to measure the density of the coexisting phases. Each of the coexisting phases was measured at least four times to determine the statistical error.

Flow small-angle light scattering experiments (SALS) measurements have been performed on a rheometrics optical analyser (ROA) that has been modified to perform SALS measurements [32]. A parallel plate geometry has been used and the temperature was controlled by means of a water bath at $298 \mathrm{~K}$. For these experiments, the scattered light is intercepted on a screen that consists of a semi-transparent paper with a beam stop. The resulting image is recorded by a CCD camera (Ikegami ICD-810P), which is mounted under the screen. The CCD camera is connected either to a frame grabber (Data Translation DT3851) or to a video recorder to collect the scattering patterns. Home built software was used to obtain intensity profiles and contourplots of the images (SALSSOFTWARE-K.U.L.).

\section{Results and discussion}

\subsection{Rheo-optical methodology to determine interfacial tension}

The determination of the interfacial tension is performed by means of a recently developed rheo-optical technique [22] 


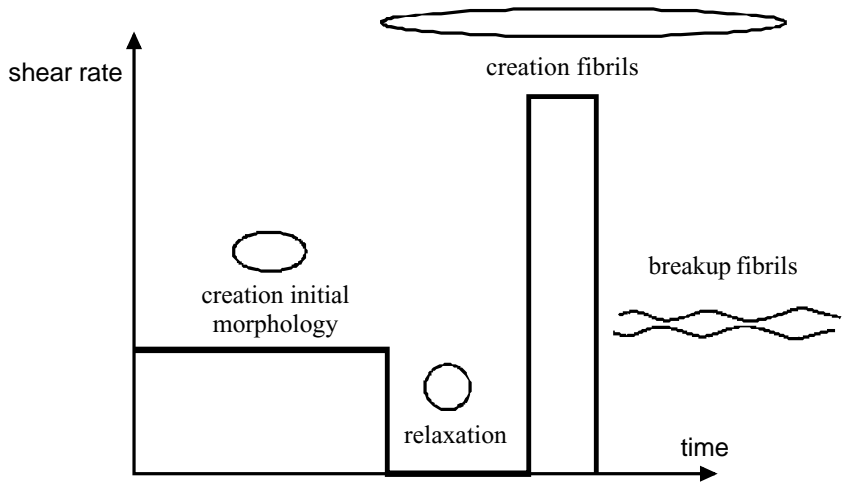

Fig. 3. Schematic flow protocol used to create the elongated fibrils.

based on Tomotika's theory of fibril break-up [33]. When a long fluid filament is present in a quiescent fluid matrix, interfacial instabilities due to thermal fluctuations will occur. These so-called Rayleigh instabilities will start to grow and will eventually disintegrate the fibril. Tomotika derived the following formula for the break-up time $t_{\mathrm{B}}$ of a Newtonian fibril immersed in a quiescent Newtonian matrix [33]:

$t_{\mathrm{B}}=\frac{2 \eta_{\mathrm{m}} R_{0}}{\Gamma \Omega} \ln \frac{\alpha_{\mathrm{B}}}{\alpha_{0}}$

where $\alpha_{\mathrm{B}}$ is the amplitude of the instability at break-up, $\alpha_{0}$ is the amplitude of the initial disturbance, $\eta_{\mathrm{m}}$ is the viscosity of the matrix material (in this case the alginate-enriched phase), $\Gamma$ the interfacial tension, $R_{0}$ is the initial radius of the undisturbed fibril that is breaking and $\Omega$ is a known function of the viscosity ratio of the mixture. Because of the dilute nature of the systems under investigation, the thermal value for $\alpha_{0}$ [34] will be used in the calculations leading to following expression for the break-up time:

$t_{\mathrm{B}}=\frac{\eta_{\mathrm{m}} R_{0}}{\Gamma \Omega} \ln \frac{10^{23} \Gamma R_{0}^{2}}{T}$

If all parameters are known, the interfacial tension can be calculated from Eq. (3) once $t_{\mathrm{B}}$ and $R_{0}$ are measured.

The experimental flow protocol to generate fibrils consists of two parts and is schematically shown in Fig. 3. First the mixture is presheared to ensure a reproducible and well-defined initial morphology. The sample is allowed to relax for $10 \mathrm{~s}$ leaving enough time for full retraction of the deformed droplets. To create elongated fibrils a high shear rate is applied. This shear rate is chosen such that the resulting capillary number (representing the ratio of the hydrodynamic stress over the interfacial stress) largely exceeds the critical capillary number.

However, rather than going through the complete process of deformation, breakup and coalescence of the droplets, the flow can be stopped during the deformation process. Moreover, conditions can be created that ensure breakup by Rayleigh instabilities [35,36]. During this disintegration, the evolution of the SALS patterns is monitored. It is known that during fibril breakup a peculiar scattering pattern emerges. It consists of two parts: central streak perpendicular to the prior flow direction is observed accompanied by secondary streaks. The intensity of these secondary streaks is increasing in time during the break-up process of the fibrils.

Mewis et al. [21] demonstrated that the evolution of these scattering patterns could be modelled on the basis of Fraunhofer diffraction theory. More interestingly, important morphological information can be deduced from the patterns. From the scattering model, a simple relation between the polar angle of the secondary streak $\left(\theta_{\mathrm{m}}\right)$ and the dominant wavelength of Rayleigh break-up $\lambda_{R}$ can be found:

$\theta_{\mathrm{m}}=\arcsin \left(\frac{\lambda_{1}}{n_{\mathrm{m}} \lambda_{\mathrm{R}}}\right)$

with $n_{\mathrm{m}}$ the refractive index of the medium and $\lambda_{1}$ the wavelength of the light. According to Tomotika's model the relationship between the dominant wavelength and the initial fibril radius $R_{0}$ is determined by the viscosity ratio through [33]:

$x_{\mathrm{m}}=\frac{2 \pi R_{0}}{\lambda_{\mathrm{R}}}$

with $x_{\mathrm{m}}$ a known function of the viscosity ratio of the blend. Hence, from the SALS patterns, $R_{0}$ can be obtained experimentally. In addition Van Puyvelde et al. [22] and Mewis et al. [21] demonstrated that the moment at which the intensity in the second streak starts to level off can be identified with the break-up time $t_{\mathrm{B}}$ of the fibrils. The levelling off or break-up point can be determined by identifying the maximal slope in the intensity versus time curve. With $t_{\mathrm{B}}$ and $R_{0}$ known, Eq. (3) can be used to obtain the interfacial tension. Various experiments can be performed varying both the applied shear rate and shearing time leading to a set of $t_{\mathrm{B}}$ and $R_{0}$ that are fitted to Eq. (3) to obtain the interfacial tension.

In order to study the effect of the location of the system in the phase diagram on the interfacial tension, systems located along four tie lines have been studied as indicated on Fig. 2. Two tie lines $\left(T_{1}\right.$ and $\left.T_{2}\right)$ were located very close to the critical point and two were located relatively far from the critical point $\left(T_{3}\right.$ and $\left.T_{4}\right)$. The phase composition, viscosity and density of the coexisting phases of the various $\mathrm{w}-\mathrm{c}-\mathrm{a}$ systems are given in Table 1.

To create a blend with a droplet matrix morphology, a prerequisite to form the elongated fibrils during the flow protocol, $1 \%$ emulsions of the caseinate-rich phase in the alginate-rich phase have been used. The low concentrations of the dispersed phase are chosen to provide sufficient transparency necessary to perform the optical measurements. The arrows presented in Fig. 2 represent the emulsions studied in this work.

Fig. 4 shows the evolution of the SALS patterns of the two-phase system located the closest to the critical point (tie line $\mathrm{T}_{1}$ ) after an interrupted start-up in shear (shear rate of $3 \mathrm{~s}^{-1}$ maintained for $3 \mathrm{~s}$ ). The corresponding microscopy images during the same flow history are shown in Fig. 5. During the deformation stage, the SALS pattern becomes highly anisotropic. This scattering pattern corresponds to 
Table 1

Phase compositions, viscosity and density of coexisting phases of the water-casein-alginate system

\begin{tabular}{|c|c|c|c|c|c|c|c|c|}
\hline \multirow[t]{3}{*}{ Tie line } & \multicolumn{4}{|c|}{ Alginate-enriched phase } & \multicolumn{4}{|c|}{ Casein-enriched phase } \\
\hline & \multicolumn{2}{|c|}{ Composition (g/l) } & \multirow[t]{2}{*}{ Viscosity (Pas) } & \multirow[t]{2}{*}{ Density $(\mathrm{g} / \mathrm{l})$} & \multicolumn{2}{|c|}{ Composition (g/l) } & \multirow[t]{2}{*}{ Viscosity (Pas) } & \multirow[t]{2}{*}{ Density $(g / l)$} \\
\hline & Caseinate & Alginate & & & Caseinate & Alginate & & \\
\hline 1 & 42.7 & 6.0 & 0.088 & $1009.0 \pm 1.0$ & 77.0 & 1.6 & 0.025 & $1013.6 \pm 1.4$ \\
\hline 2 & 24.2 & 8.9 & 0.120 & $1008.0 \pm 0.8$ & 87.0 & 0.8 & 0.041 & $1014.3 \pm 1.3$ \\
\hline 3 & 4.47 & 12.4 & 1.220 & $1007.5 \pm 0.7$ & 131.0 & 0.5 & 0.453 & $1030.3 \pm 1.0$ \\
\hline 4 & 2.0 & 14.5 & 1.920 & $1007.0 \pm 0.9$ & 144.3 & 0.5 & 1.340 & $1040.0 \pm 1.2$ \\
\hline
\end{tabular}

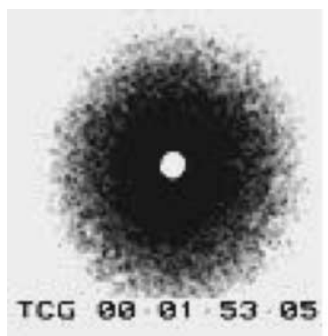

After preshear

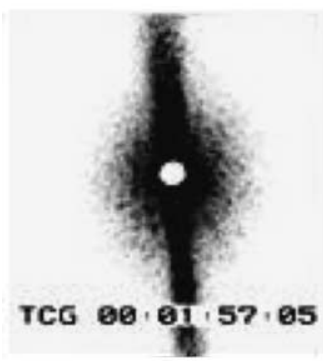

After stopping

Flow: $1.0 \mathrm{~s}$

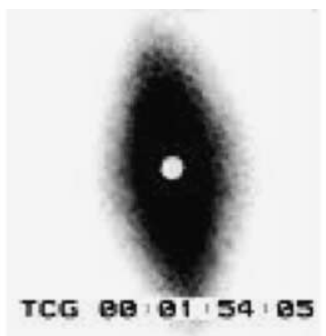

Shear : $1.0 \mathrm{~s}$

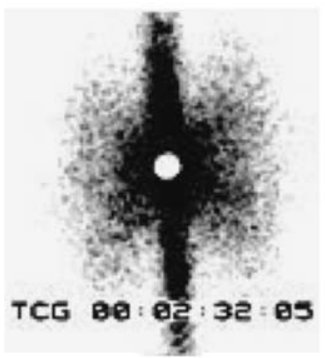

$36 \mathrm{~s}$

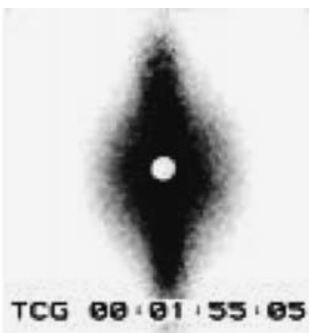

$2.0 \mathrm{~s}$

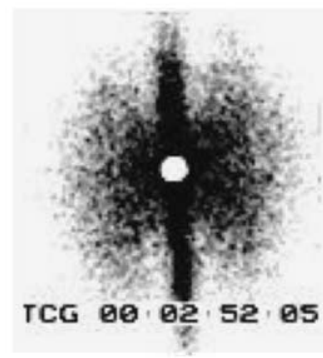

$56 \mathrm{~s}$

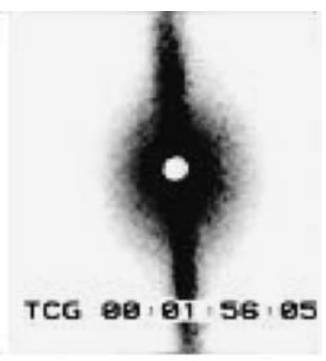

$3.0 \mathrm{~s}$ (moment of stopping flow)

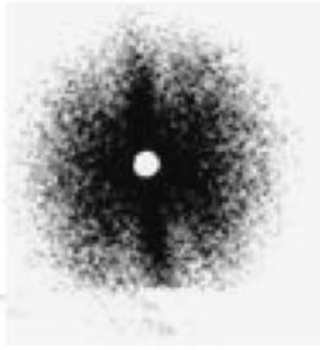

$78.0 \mathrm{~s}$

Original flow direction

Fig. 4. Evolution of the SALS patterns for an emulsion along $\mathrm{T}_{1}$ after an interrupted start-up to a shear rate of $3 \mathrm{~s}^{-1}$, interrupted after $3 \mathrm{~s}$.

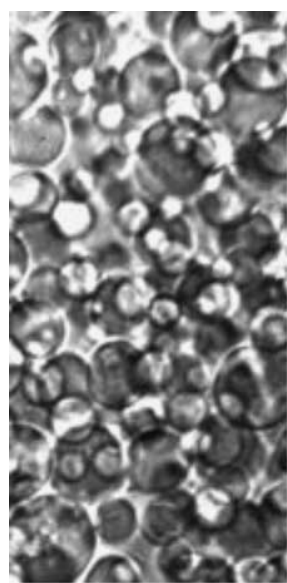

(A)

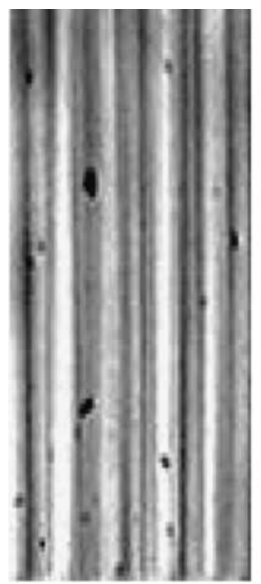

(B)

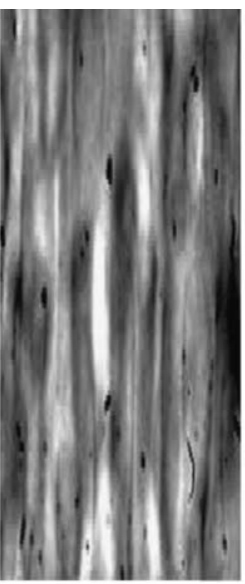

(C)

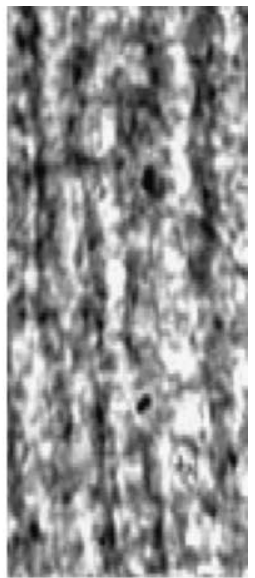

(D)

Fig. 5. Evolution of the microscopy images, same conditions as in Fig. 4: (A) before application of the high shear rate; (B) moment of interrupting the flow $\left(3 \mathrm{~s}\right.$ at $\left.3 \mathrm{~s}^{-1}\right)$; (C) $36 \mathrm{~s}$ after stopping the flow; (D) $76 \mathrm{~s}$ after stopping the flow. 
highly elongated fibrils oriented in the flow direction as is shown in Fig. 5B. After stopping the flow, a secondary streak appears in the SALS images, indicative of the occurrence of Rayleigh instabilities. It should be noticed that the time, necessary to observe the development of the secondary streak, is rather long compared with the time-scales usually encountered in synthetic polymer blends [22]. It can be noticed that the intensity in the secondary maximum is increasing with time and finally levels off when the fibrils are transformed to a string of separated droplets. From the analysis of this intensity as a function of time, the breakup time of the fibrils can be determined. During the disintegration process, the wavelength of the disturbance remains constant but its amplitude increases until the fibril finally breaks into a series of smaller droplets (Fig. 5D). Fig. 6 shows the intensity in the secondary streak as a function of time ( $t=0$ corresponds to the interruption of the flow). The analysis of the SALS patterns is performed by the method explained above and elsewhere $[9,21,22]$ leading - for the experiments represented in Fig. 4- to a fibril radius of $0.9 \mu \mathrm{m}$ and a breakup time of $46 \mathrm{~s}$.

With the break-up times and the values of $R_{0}$ obtained from the SALS experiments, Eq. (3) can be used to obtain a value of the interfacial tension for the systems located along the four tie lines. For the system located along $\mathrm{T}_{1}$, the interfacial tension amounts to $1.2 \times 10^{-8} \pm 0.2 \times 10^{-8} \mathrm{~N} / \mathrm{m}$. The values for the other systems studied are given in Table 2 . The rheo-optical method is capable of measuring these extremely low values of the interfacial tension, a value which would challenge most of the more classical interfacial tension methods. These results extend the applicability of the technique since it was originally developed for blends of synthetic polymers where the interfacial tension is of the order of $10^{-3} \mathrm{~N} / \mathrm{m}$.

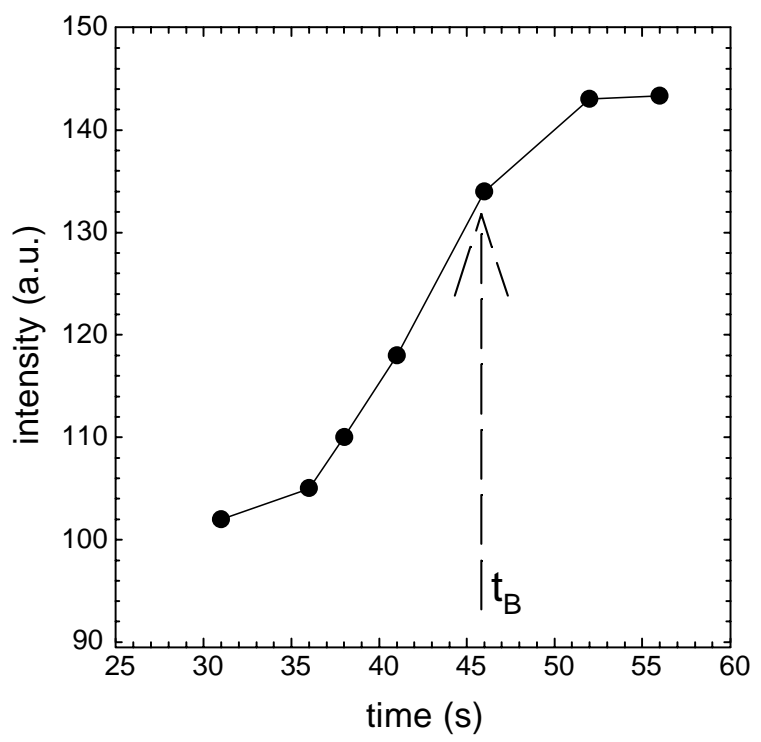

Fig. 6. Intensity of the secondary streak as a function of time (same conditions as in Fig. 4).
Table 2

Interfacial tension for the systems located on the different tie lines

\begin{tabular}{lcl}
\hline Tie line & Density difference $(\mathrm{g} / \mathrm{l})$ & Interfacial tension $(\mathrm{N} / \mathrm{m})$ \\
\hline 1 & 4.6 & $1.2 \times 10^{-8} \pm 0.2 \times 10^{-6}$ \\
2 & 6.3 & $2.1 \times 10^{-8} \pm 0.2 \times 10^{-6}$ \\
3 & 22.5 & $1.2 \times 10^{-6} \pm 0.2 \times 10^{-6}$ \\
4 & 33.0 & $5.2 \times 10^{-6} \pm 0.2 \times 10^{-6}$ \\
\hline
\end{tabular}

\subsection{Scaling of the interfacial tension}

In order to compare with theoretical predictions, the interfacial tension has been evaluated compared to the density difference between the coexisting phases. Obviously, at the critical point, the density difference $\Delta \rho$ between the coexisting phases equals 0 . Hence the density difference is an indication of the distance of the system from the critical point. This approach is similar to the approach of Scholten et al. who used a spinning drop method to measure the interfacial tension of an aqueous gelatin/dextran mixture [10]. However, the values of the interfacial tension reported here are an order of magnitude smaller than the ones reported by Scholten et al. The distance from the critical point for the system studied by Scholten et al. is similar to the location of system $T_{1}$. To compare them on a relative scale, the density difference $\Delta \rho$ can be normalised by the density at the critical point $\rho_{\text {crit }} . \rho_{\text {crit }}$ can be estimated, based on Table 1 , as $1010 \mathrm{~g} / \mathrm{l}$, yielding a relative distance smaller than 0.005 which is similar to the values reported by Scholten et al. However, sodium iodide was added in their work to achieve a small density difference between the coexisting phases that was a prerequisite to make use of their spinning drop method, a drawback that is not present with this technique.

In Fig. 7 the interfacial tension is plotted as a function of the density difference $(\Delta \rho)$ between the coexisting phases. The interfacial tension close to the critical point is very small $\left(1.2 \times 10^{-8} \mathrm{~N} / \mathrm{m}\right)$ and increases to a value

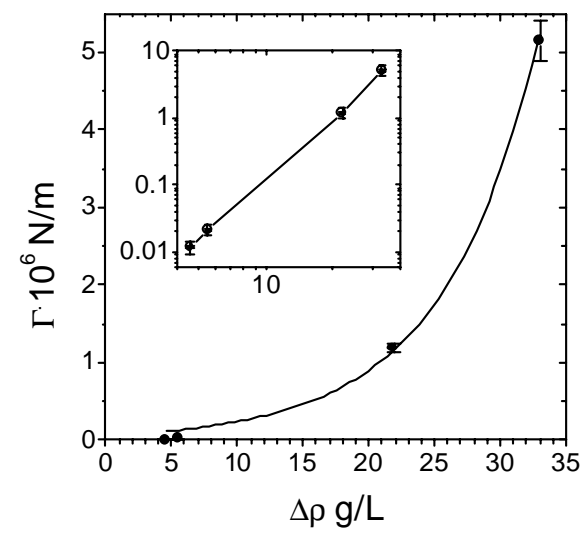

Fig. 7. Interfacial tension of the systems along four tie lines as a function of the density difference. The full line is the best fit to the mean field scaling relation: $\Gamma=a(\Delta \rho)^{b}$. The insert shows the scaling plotted logarithmically. 
of $5.2 \times 10^{-6} \pm 0.15 \times 10^{-6} \mathrm{~N} / \mathrm{m}$ farther from the critical point $\left(\mathrm{T}_{4}\right)$. The latter value for the mixture located on the phase diagram far from the critical point is in reasonable agreement to that obtained by Capron et al. $\left(8 \times 10^{-6} \mathrm{~N} / \mathrm{m}\right)$ for a similar system [25] and similar to that reported by Guido et al. [20] $\left(8.8 \times 10^{-6} \mathrm{~N} / \mathrm{m}\right)$ for a sodium caseinate (6 wt.\%)-sodium alginate (1.0 wt.\%) system located far from the binodal.

As can be seen in Fig. 7, the dependence of $\Gamma$ on $\Delta \rho$ is non-linear. The data were fitted to the relation: $\Gamma=a(\Delta \rho)^{b}$, for which $b=3.1 \pm 0.3$ was found as best-fit parameter. The scaling exponent of $3.1 \pm 0.3$ is very close to that (3.0) predicted by mean-field theory [37].

A similar scaling exponent $(2.7 \pm 0.3)$ was obtained by Scholten et al. [10] for the gelatin-dextran-1 M NaI system. This scaling exponent is important since it allows to describe the interfacial tension in phase-separated biopolymer mixtures at different conditions provided the knowledge of one reference interfacial tension and the knowledge of the densities of the coexisting phases. This interfacial tension can then be used to describe for instance the phase separation or the morphology development during flow.

\section{Conclusions}

Using a rheo-optical methodology, based on SALS measurement, the interfacial tension of a two-phasic watersodium caseinate-sodium alginate system has been determined as a function of the location on the phase diagram. The interfacial tension close to the critical point was $1.2 \times$ $10^{-8} \mathrm{~N} / \mathrm{m}$, and it increased to values of $5 \times 10^{-6} \mathrm{~N} / \mathrm{m}$ farther from the critical point. The rheo-optical methodology is very well suited to capture these very low values of the interfacial tension; the measurement window spans interfacial tensions ranging from $10^{-3}$ up to $10^{-8} \mathrm{~N} / \mathrm{m}$, which is hard to achieve with conventional techniques. The relation between interfacial tension and density difference between the phases has been determined and was compared with theoretical predictions. The experimental scaling exponent was in good agreement with critical mean-field values.

\section{Acknowledgements}

P.V.P. is indebted to the FWO-Vlaanderen for a postdoctoral fellowship. Y.A. thanks the Research Council of the K.U. Leuven for a senior fellowship. This research has been partially funded by a GOA project (1998/06 and 2003/06) from the Research Fund K.U. Leuven.

\section{References}

[1] Tolstoguzov V. In: Roos YH, Leslie RB, Liiford PJ, editors. Water management in the design and distribution of quality foods. Lancaster, Basel: Technomic Publishing Company Inc.; 1999. p. 199-233.

[2] Antonov YA, Grinberg VY, Zhuravskaya NA, Tolstoguzov VB. J Texture Stud 1980;11:199.

[3] Antonov YA, Soshinsky AA. Int J Biol Macr 2000;27:279.

[4] Harding SE, Hill SE, Mitchell JR. Biopolymer mixtures. Nottingham: Nottingham University Press; 1995.

[5] Tolstoguzov VB. In: Mitchel IR, Ledward DA, editors. Functional properties of food macromolecules. London: Elsevier Applied Science; 1986. p. 385.

[6] Antonov YA, Grinberg VY, Tolstoguzov VB. Starke 1975;27:424.

[7] Antonov YA, Grinberg VY, Tolstoguzov VB. Polym Sci USSR 1976;18:566 [English translation].

[8] Albertsson P-A. Partitioning in aqueous two-phase systems: theory, methods, uses and applications to biotechnology. Orlando: Academic Press; 1985.

[9] Van Puyvelde P, Antonov YA, Moldenaers P. Food Hydrocolloids 2002;5:795.

[10] Scholten E, Tuinier R, Tromp RH, Lekkerkerker HNW. Langmuir 2002;18:2234.

[11] Antonov YA, Soshinsky AA. Int J Biol Macr 2000;27:279.

[12] Kasapis S, Morris ER, Norton IT, Gidley MJ. Carbohydr Polym 1999;21:249.

[13] Gottschaalk M, Linse P, Piculell L. Macromolecule 1998;31:8407.

[14] Khokhlov AR, Nyrkova IA. Macromolecule 1992;25:1493.

[15] Tolstoguzov VB. Int Rev Cytol 2000;192:3.

[16] Antonov YA. Appl Biochem Microbiol 2000;36:325.

[17] Funke Z, Schwinger C, Adhikari R, Kressler J. Macromol Mater Eng 2001;286:744.

[18] Tucker CL, Moldenaers P. Ann Rev Fluid Mech 2002;34:177.

[19] Stokes JR, Wolf B, Frith WJ. J Rheol 2001;45:173.

[20] Guido S, Simeone M, Alfani A. Carbohydrate Polym 2002;48:143.

[21] Mewis J, Yang H, Van Puyvelde P, Moldenaers P, Walker LM. Chem Eng Sci 1998;53:2231.

[22] Van Puyvelde P, Yang H, Mewis J, Moldenaers P. J Coll Int Sci 1998;299:84.

[23] Whistler RL. Industrial gums, 2nd ed. New York: Academic Press; 1973.

[24] Swaisgood HE. In: Fox PF, editor. Advanced dairy chemistry. 1: Proteins. London: Elsevier Applied Science; 1992.

[25] Capron I, Costeux S, Djabourov M. Rheol Acta 2001;40:441.

[26] Antonov YA, Grinberg VY, Tolstoguzov VB. Nahrung 1979;23:207.

[27] Antonov YA, Grinberg VY, Tolstoguzov VB. Nahrung 1979;23:597.

[28] Antonov YA, Grinberg VY, Tolstoguzov VB. Nahrung 1979;23:847.

[29] Koningsveld R, Staverman A. J Polym Sci 1968;A2:305.

[30] Polyakov VI, Grinberg VY, Tolstoguzov VB. Polym Bull 1980;2:760.

[31] Albertsson PÅ. Partition of cell particles and macromolecules, 3rd ed. New York: John Wiley \& Sons; 1986.

[32] Yang H, Zhang H, Moldenaers P, Mewis J. Polymer 1998;39:5731.

[33] Tomotika S. Proc R Soc London 1936;A150:322.

[34] Kuhn W. Kolloid Z 1953;132:94.

[35] Stone HA, Leal LG. J Fluid Mech 1989;198:399.

[36] Van Puyvelde P, Moldenaers P, Mewis J. Phys Chem Chem Phys 1999;1:2505.

[37] Rowlinson JS, Widon B. Molecular theory of capillarity. Oxford, UK: Clarendon Press; 1984. 